\title{
JORNADA LEAN: O USO DE GAMIFICAÇÃO COMO MOTIVAÇÃO PARA O APRENDIZADO NO ENSINO REMOTO
}

DOI: 10.37702/2175-957X.COBENGE.2021.3547

SANDRA RUFINO - ssrufino@yahoo.com.br

Universidade Federal do Rio Grande do Norte

Avenida Gustavo Guedes 1789

59078-380 - Natal - RN

Júlia Lúcio Bezerra Ferreira - julialuciopj@gmail.com

Universidade Federal do Rio Grande do Norte

Rua Timbaúba, Nova Parnamirim,, 202202

59152-490 - Parnamirim - RN

Resumo: Com a crise sanitária ocasionada pelo Covid 19, as universidades federais brasileiras diminuíram a duração dos períodos acadêmicos e utilizaram-se de um sistema de ensino remoto, acarretando a diminuição no engajamento dos alunos nas aulas e nas notas. Devido os protocolos de segurança orientados pela Organização Mundial de Saúde os alunos da disciplina "Gestão de Sistemas Produtivos 2" não puderam mais realizar os trabalhos práticos que eram feitos anteriormente. Após analisar as opiniões dados pelos alunos no ano anterior, foi realizada a pesquisa exploratória que iniciou com estudo bibliográfico e em seguida foi planejada e executada a Jornada de melhoria contínua, jogo que foi elaborado com o intuito de para tornar o processo de estudo mais leve, divertido e efetivo, mesmo com aulas assíncronas. A jornada foi composto por quatro categorias de atividades que ao serem completadas corretamente rendiam aos alunos pontuações e ao final do semestre os cinco alunos com as melhores colocações na classificação ganhavam notas extras. Os resultados demonstraram um bom rendimento da turma, além de aceitação e satisfação dos alunos por participarem do jogo.

Palavras-chave: Gamificação. Ensino remoto. Metodologias ativas. Covid19. 


\section{JORNADA LEAN: O USO DE GAMIFICAÇÃO COMO MOTIVAÇÃO PARA O APRENDIZADO NO ENSINO REMOTO}

\section{INTRODUÇÃO}

O curso de Engenharia de Produção em uma universidade localizada no nordeste brasileiro, adota estratégias de aprendizado ativo e a aplicação de diversos trabalhos práticos durante a trajetória acadêmica dos alunos. A disciplina profissionalizante Gestão de Sistemas Produtivos II (GSP2), que trata sobre produção enxuta, precisou se adaptar à realidade de ensino remoto, experiência que será relatada neste artigo. Essa disciplina promove com os alunos o desenvolvimento de trabalhos práticos com o método de aprendizado baseado em problemas (Problem Based Learning - PBL) em organizações locais (privadas, públicas ou terceiro setor), de modo que os alunos iam a campo e implantavam ferramentas da metodologia Lean.

Com o advento da crise sanitária causada pela pandemia do coronavírus 19 em março de 2020 as aulas foram suspensas nas universidades. A universidade optou por realizar um período experimental de aulas no formato de ensino remoto e depois retornar o período letivo 2020.1 em outubro do mesmo ano. Por causa dos protocolos de segurança orientados pela Organização Mundial de Saúde (OMS) os alunos foram impedidos de realizarem atividades práticas nas organizações e foram submetidos ao ensino $100 \%$ remoto e com períodos letivos encurtados (de 18-20 para 13-14 semanas). Essa nova realidade reduziu a interação dos alunos durante aulas de momento síncrono (impactadas pela distância e pela redução do número de aulas expositivas) sobrecarregou-os de trabalhos acadêmicos assíncronos, o que impactou em queda nas notas e no aumento dos índices de trancamentos de matérias.

Com o objetivo de mitigar os impactos negativos apresentados no contexto relatado para o período 2020.1, a docente e os monitores da disciplina GSP2, após analisarem os resultados e feedback dos alunos sobre as estratégias de aprendizado ativo adaptado para o ensino remoto, decidiram implementar para 2020.2 a aplicação de gamificação durante o curso da disciplina. O objetivo foi motivar os alunos utilizando-se de elementos da dinâmica dos jogos como forma de motivar e aprofunda os estudos da matéria (ALVES; MINHO; DINIZ, 2014). Este artigo tem por objetivo descrever essa experiência e os tópicos estão organizados nessa introdução, seguidos da metodologia, na descrição da ação e suas atividades, resultados e considerações finais.

\section{METODOLOGIA}

A primeira etapa do trabalho foi o levantamento e análise de dados. Durante o semestre 2020.1 foram elaborados dois questionários para que os alunos respondessem sobre a experiência e dificuldades de cursar a disciplina por meio do ensino remoto. A partir das respostas coletadas, foi realizado um estudo bibliográfico buscando achar uma estratégia de ensino que pudesse mitigar alguns dos obstáculos enfrentados pelos alunos.

Em seguida, houve o planejamento inicial, construção de um documento elucidativo para que os alunos ficassem cientes de como funcionaria o semestre letivo e então começou a aplicação da gamificação, de modo que durante o semestre as atividades foram sendo elaboradas e em seguida experimentadas com os alunos, realizando avaliação sobre quais características de tais atividades e materiais mais culminavam em maior participação por parte dos discentes. Com base no explicitado, podemos caracterizar o trabalho como 
exploratório descritivo com abordagem qualitativa, visto que teve como objetivo resolver um problema específico, sem estrutura de execução bem estruturada, levando em consideração bibliografias e entrevistas com os sujeitos a qual a metodologia foi aplicada.

\section{JORNADA DA MELHORIA CONTÍNUA}

Desenvolveu-se o jogo denominado de "Jornada da Melhoria Contínua", nome que faz alusão a um dos princípios da filosofia estudada. O jogo possui quatro atividades principais, cada uma com vários pontos de contato e valendo uma pontuação pré-definida. A recompensa da jornada foi estipulada como nota extra para os cinco primeiros colocados, os alunos com maior pontuação, ao final do semestre letivo receberam $20,15,12,8$ e $5 \%$, de pontuação extra, respectivamente do primeiro ao quinto classificado a ser acrescido na nota conquistada pelo discente na unidade de sua escolha (são 3 unidades/semestre). A "Jornada da Melhoria Contínua" foi composta pelas atividades (detalhadas mais à frente): 1) Dica Lean da Semana; 2) Webquests; 3) Lean na minha casa e; 4) Formulários de autoavaliação e diagnóstico.

Jogos aplicados em um contexto pedagógico devem ser capazes de deixar os jogadores muito envolvidos (RUOHOMÄKI, 1995) e esse engajamento foi observado na Jornada da Melhoria Contínua, visto que todos os trinta e nove alunos da turma participaram de pelo menos três das quatro categorias de atividades propostas.

Para apresentar o jogo aos alunos, foi desenvolvido o "Guia de Sobrevivência a GSP2 no Ensino Remoto", que explicava, de maneira lúdica e com identidade visual chamativa, todas as etapas e regras da trilha, e utilizava como enunciador da mensagem o Mestre Ohno, fazendo referência ao criador da Filosofia Lean, Taiichi Ohno.

Figura 1 - Guia de Sobrevivência a GSP2 no ensino Remoto

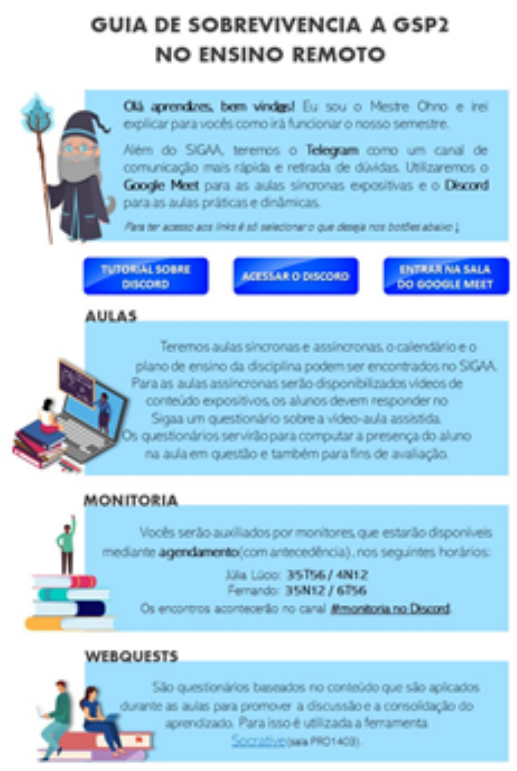

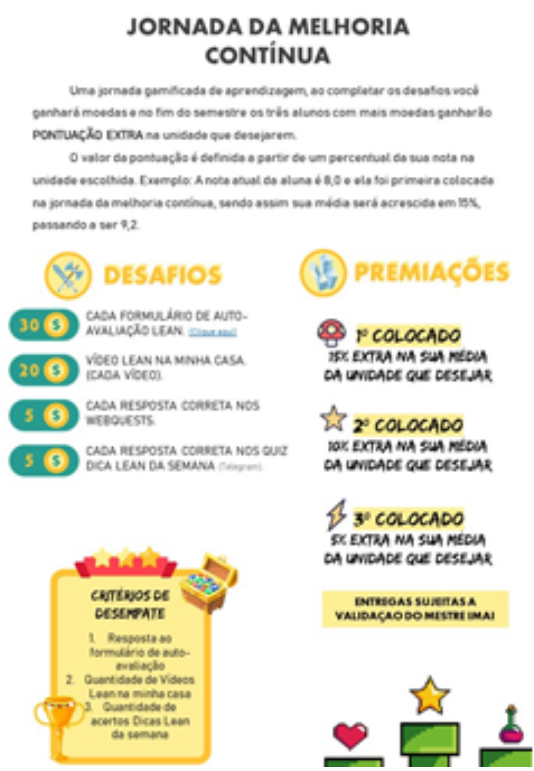

Fonte: Autoria própria, 2021

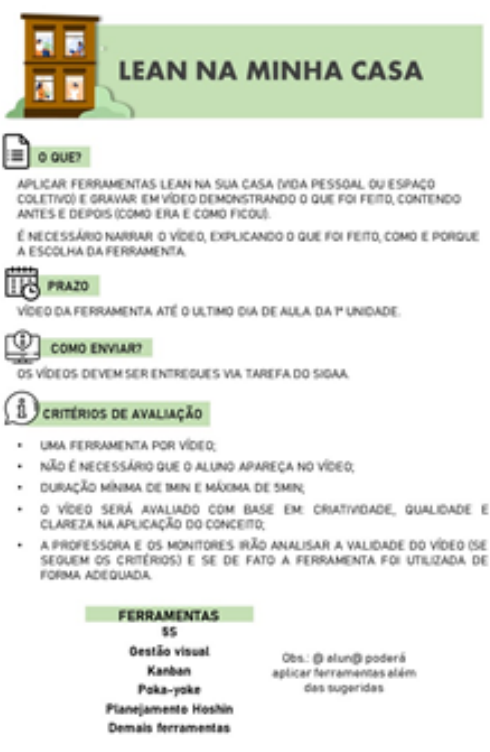

\section{ATIVIDADES DA JORNADA}

\subsection{Dica Lean da Semana}

O Dica Lean da Semana partiu do desejo de estimular os alunos a explorarem conteúdos além dos materiais produzidos pela equipe de monitores e professora. Sendo assim, foram disponibilizados semanalmente, pelo aplicativo Telegram, vídeos ou artigos 
para os alunos, juntamente com quizzes indagando-os sobre o tema explorado, de modo que a cada resposta correta foram atribuídos pontos para a classificação na jornada.

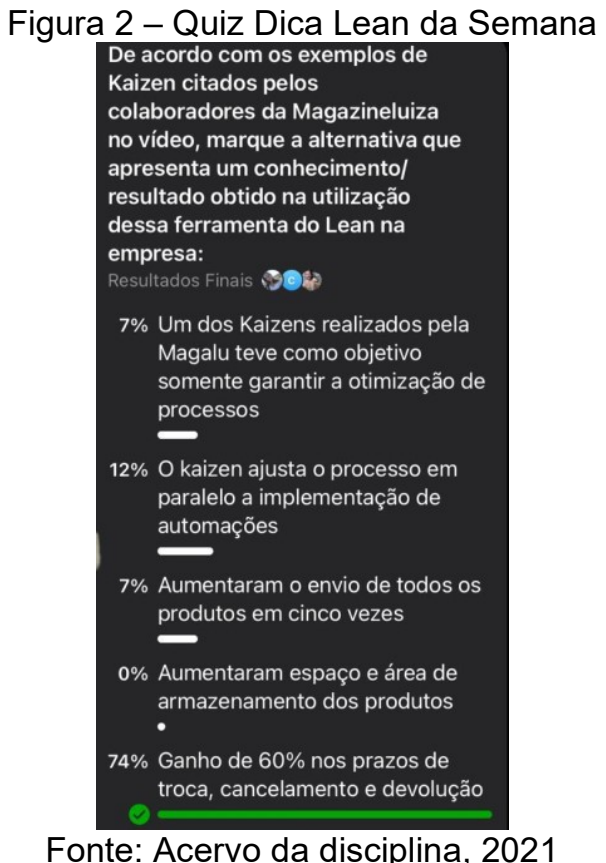

\subsection{Webquest}

Os webquests objetivaram incentivar os alunos a seguirem o cronograma de estudos assíncronos proposto e orientado pela docente. Nesse período remoto foi aplicada a metodologia ativa de sala de aula invertida, de modo que os alunos estudavam os vídeos e apostilas desenvolvidas pela professora e depois de uma semana, tinham aula síncrona para a retirada de dúvidas e análise de exemplos de casos reais. Para essas aulas foram aplicados questionários sobre o tópico da semana, utilizando o programa Socrative, de forma que, para obter as pontuações seria necessário acertar as questões, e para isso os alunos foram encorajados a estudar conforme o planejamento da disciplina.

\begin{tabular}{|c|c|c|c|c|c|c|}
\hline \multirow[b]{2}{*}{ NOME $\triangle$} & \multirow{2}{*}{ NOTA \% } & & \multirow[b]{2}{*}{5} \\
\hline & & 1 & 2 & 3 & 4 & \\
\hline Clara & $\checkmark 73 \%$ & $\checkmark B$ & $\checkmark D$ & $\times C$ & $\times A$ & $\checkmark C$ \\
\hline Daniel & $\checkmark 100 \%$ & $\checkmark B$ & $\checkmark D$ & $\checkmark D$ & $\checkmark C$ & $\checkmark C$ \\
\hline Dilana & $\checkmark 82 \%$ & $\checkmark B$ & $\checkmark D$ & $\times A$ & $\checkmark C$ & $\checkmark C$ \\
\hline Eloísa & $\checkmark 82 \%$ & $\checkmark B$ & $\checkmark D$ & $\checkmark D$ & $\checkmark C$ & $\checkmark C$ \\
\hline Felipe alves & $\checkmark 82 \%$ & $\checkmark B$ & $\checkmark D$ & $\checkmark D$ & $\checkmark C$ & $\checkmark C$ \\
\hline
\end{tabular}

Fonte: Acervo da disciplina, 2021

\subsection{Formulário de Autoavaliação}

Outra atividade proposta nessa jornada foi o formulário de auto-avaliação e diagnóstico, aplicados no início do semestre acerca do entendimento dos tópicos a serem estudados na disciplina, que buscaram auxiliar na construção pragmática dos conteúdos extras disponibilizados, bem como identificar o que deveria ter mais foco durante explanação teórica em sala de aula. Esse documento também serviu para o diagnóstico das condições, tanto psicológicas quanto de infraestrutura, para o modelo de ensino remoto 
no contexto da pandemia, que permitiu estabelecer flexibilidade em cronogramas e/ou melhor acolhimento dos alunos com dificuldades.

Figura 4 - Formulário de autoavaliação

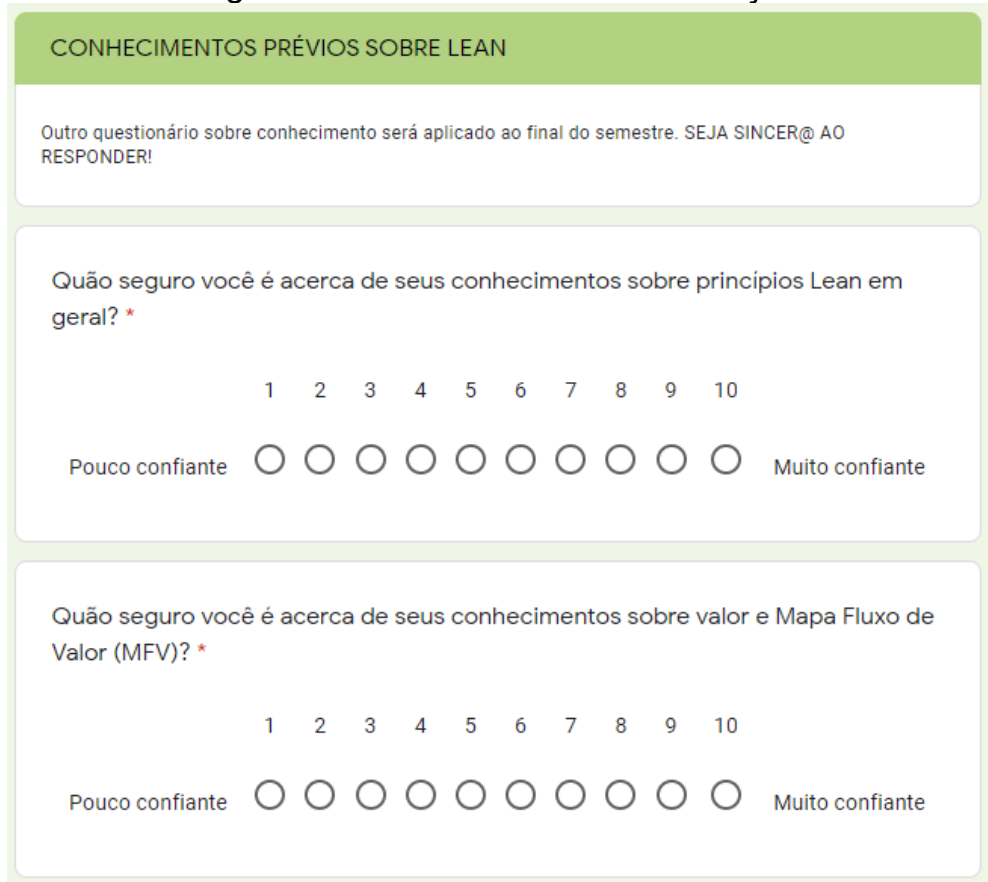

Fonte: Acervo da disciplina, 2021

\subsection{Lean na Minha Casa}

O Lean na minha casa consiste nos alunos documentarem a aplicação de uma das ferramentas da Filosofia Lean em sua rotina ou seu lar, por meio de vídeos entre 1 e 5 minutos, possibilitando que eles desenvolvam a capacidade crítica e o diagnóstico de necessidade de melhoria, bem como exercitem as ferramentas aprendidas na disciplina de maneira prática, já que a aplicação em campo (empresas parceiras da universidade) não era uma possibilidade, devido à situação de pandemia vivida nos anos 2020 e 2021 . Esse foi o único desafio da trilha que a pontuação não era fixa, estabeleceu-se um limite máximo de 20 pontos por vídeo, mas os pontos recebidos pelos alunos variavam segundo a qualidade da implementação da ferramenta e da qualidade da explicação do motivo de sua aplicação.

Figura 5 - Vídeos Lean na Minha Casa

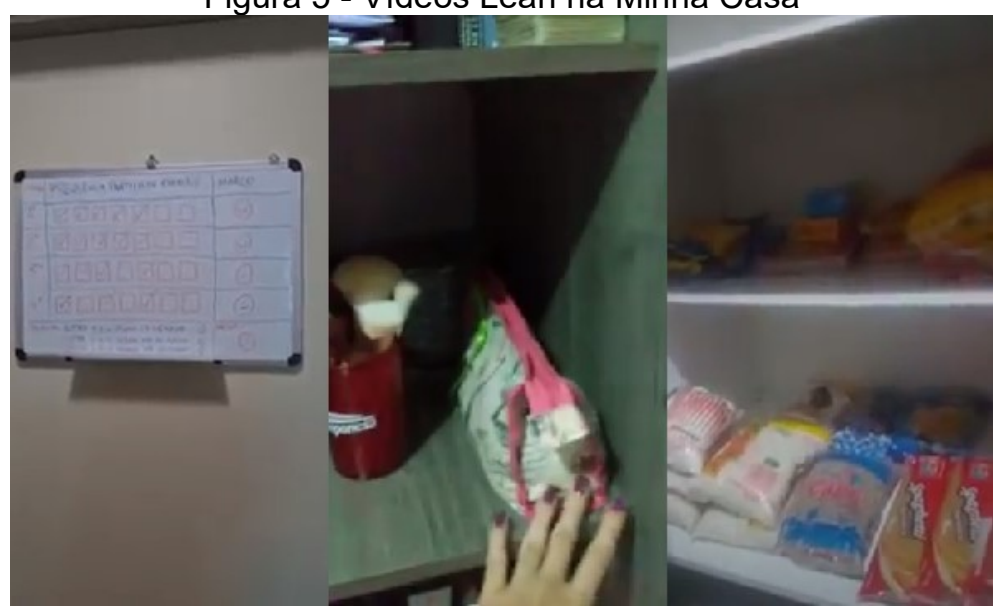

Fonte: Acervo da disciplina, 2021 


\section{$5 \quad$ MECANISMOS DE JOGOS UTILIZADOS}

Existem diversas técnicas de gamificação, entre elas temos regras, pontos, feedbacks, rankings e conquistas (KLOCK et al., 2014), tais elementos foram aplicados na Jornada da melhoria contínua e estão descritos a seguir:

\subsection{Regras}

Foram inseridas no "Guia de Sobrevivência: GSP2 no Ensino Remoto", informações que descreviam sobre o que era cada uma das missões a serem cumpridas, a pontuação que cada uma representava, quais os prêmios da jornada e critérios de desempate entre jogadores com a mesma pontuação no fim do período.

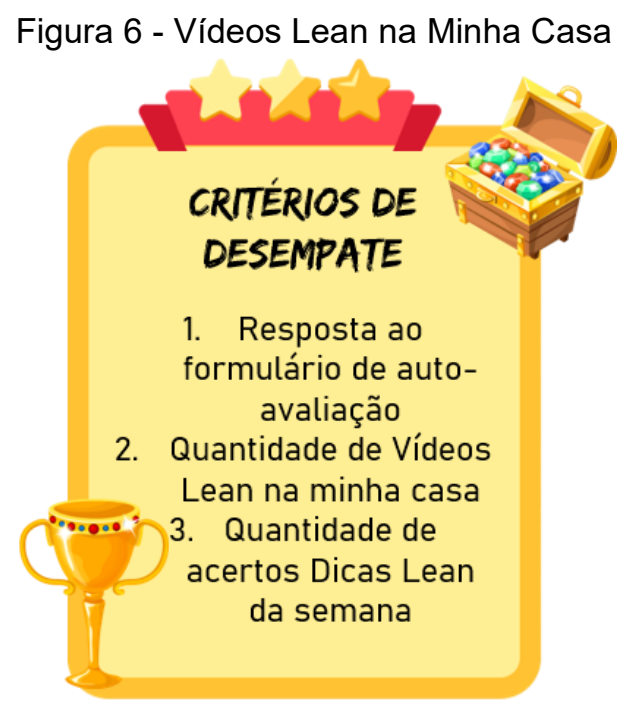

Fonte: Autoria própria, 2021

\subsection{Pontuação}

Todos os jogadores começaram a jornada com zero pontos e foram ganhando pontos a partir da realização das missões propostas, não havendo ações que os fizessem perder os pontos já conquistados.

Figura 7 - Pontuação por Desafio

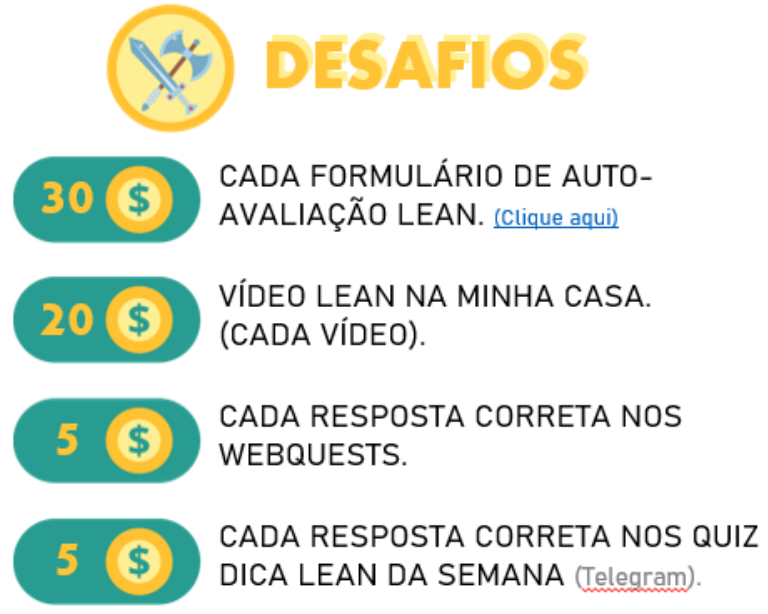

Fonte: Autoria própria, 2021 


\subsection{Feedback}

As ferramentas utilizadas eram programadas para dar feedbacks instantâneos (Telegram e Socrative) para os alunos sobre o acerto ou não do conteúdo e consequentemente a pontuação obtida. Apenas no Lean na Minha Casa, que exigiu um processo maior de análise dos vídeos., as devolutivas foram enviadas após sete dias com os comentários da docente e dos monitores, que fizeram a avaliação técnica de cada ferramenta aplicada, que é apresentada nos vídeos.

\subsection{Ranking}

Nenhuma tarefa era obrigatória, eles realizavam se quisessem, sendo estimulados a realizarem apenas para subir posições no ranking, que era atualizado a cada quinze dias e publicado para o grupo da turma no Telegram.

\begin{tabular}{|c|c|c|}
\hline Posição & Jogador & Pontuação \\
\hline 1 & JULIA & 170 \\
\hline 2 & (10) ANA LUIZA & 165 \\
\hline 3 & (0) DILANA & 163 \\
\hline 4 & $\aleph$ TAINE & 160 \\
\hline 5 & 1 calo & 155 \\
\hline
\end{tabular}

Fonte: Autoria própria, 2021

\subsection{Conquistas}

A recompensa definida para os primeiros colocados da jornada são pontos extras na nota da disciplina, sendo percentual em relação a nota já obtida pelo discente, de modo que quanto maior for a sua nota por meio de provas e trabalhos nas unidades, maior será a pontuação extra recebida.

Figura 9 - Premiações
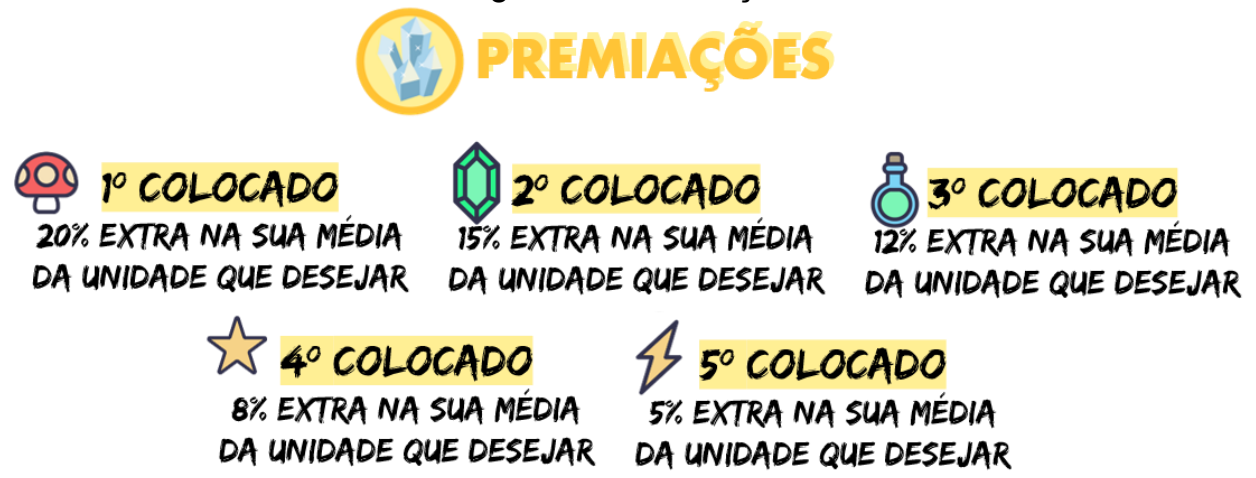

Fonte: Autoria própria, 2021

\section{CONSIDERAÇÕES FINAIS}

Através de esforços conjuntos entre monitores e docente, foi desenvolvido um jogo objetivando incentivar os discentes a se tornarem protagonistas de seu aprendizado no curso de engenharia de produção, o que se tornou ainda mais essencial em um momento 
em que o modelo de ensino remoto foi adotado pelas instituições de ensino superior do país.

A utilização de recursos de jogos poderia ser aprimorada, incluindo o alcance de níveis, por parte do aluno, durante a trilha do conhecimento estabelecida na jornada, aumentando a dificuldade com o tempo. Outro ponto que também poderia trazer aperfeiçoamento, seria a utilização de narrativa ficcional durante a apresentação de cada uma das atividades, para que os alunos se sintam atraídos não só pelo prazer de jogar, mas também tenham sensações de adrenalina, competição e aventura, mantendo as emoções motivadoras.

A aplicação da Jornada da Melhoria Contínua e todos os seus desafios se mostrou muito positiva no processo ensino-aprendizagem. Ao serem questionados sobre qual nota dariam para essa estratégia de ensino, os alunos atribuíram uma nota média de 4,27 de um máximo de 5 pontos, demonstrando sua adesão e importância no que tange à participação e à dedicação dos discentes para com a disciplina ministrada, além do para o aprendizado adquirido e apreendido.

Além dos benefícios já citados, a Jornada possibilitou a mensuração contínua acerca do entendimento e da absorção dos conhecimentos do curso por parte dos estudantes, permitindo que as aulas e atividades fossem adaptadas para os assuntos que os alunos têm mais dificuldade.

\title{
REFERÊNCIAS
}

ALVES, Lynn Rosalina Gama; MINHO, Marcelle Rose da Silva; DINIZ, Marcelo Vera Cruz. Gamificação: diálogos com a educação. In: FADEL, Luciane Maria et al. (Org.). Gamificação na educação. São Paulo: Pimenta Cultural, 2014, p. 74-97.

GANGA, Gilberto. Metodologia científica e trabalho de conclusão de curso (TCC): um guia prático de conteúdo e forma. Universidade Federal de São Carlos - UFSCar, 2011.

KLOCK, Ana Carolina Tomé. et al. Análise das técnicas de Gamificação em Ambientes Virtuais de Aprendizagem. Revista Novas Tecnologias na Educação, v. 12 n. 2, 2014.

RUOHOMÄKI, V. Viewpoints on learning and education with simulation games. In: Riis J.O. (eds) Simulation games and learning in production management. APMS 1995. IFIP Advances in Information and Communication Technology. Springer, Boston, MA, 1995.

\section{THE LEAN MANUFACTURING JOURNEY: IMPLEMENTING GAMIFICATION AS TOOL FOR MOTIVATING STUDENTS IN A REMOTE TEACHING SCENARIO}

\begin{abstract}
Due to the Covid-19 sanitary crisis, Brazilian federal universities have reduced the length of academic periods and used a remote education system, leading to decreased grades and student engagement. Following the safety protocols guided by the World Health Organization, students of the discipline "Management of Productive Systems 2" could not do the practical work that was done previously. After analyzing the feedback given by the students in the previous year, exploratory research was carried out, which started with a bibliographic study, and then the Continuous Improvement Journey was planned and executed. It is a game designed to make the learning process lighter, fun, and practical,
\end{abstract}


even with asynchronous classes. The game consisted of four types of activities that, when completed correctly, earned students' scores, and at the end of the semester, the five students with the best scores in the ranking earned extra grades. The results showed that participating in the game brought an excellent performance and a high satisfaction rate.

Keywords: Gamification. Remote teaching model. Active teaching methodologies. Covid 19 pandemic. 